\title{
La minorité acadienne au Canada atlantique
}

\author{
Rodolphe M.J. Toussaint
}

\section{OpenEdition}

Journals

Édition électronique

URL : http://journals.openedition.org/ries/2958

DOI : 10.4000/ries.2958

ISSN : 2261-4265

\section{Éditeur}

Centre international d'études pédagogiques

\section{Édition imprimée}

Date de publication : 1 mars 1998

Pagination : 97-106

ISBN : 9771254459005

ISSN : 1254-4590

Référence électronique

Rodolphe M.J. Toussaint, «La minorité acadienne au Canada atlantique », Revue internationale d'éducation de Sèvres [En ligne], 17 | 1998, mis en ligne le 19 avril 2013, consulté le 01 mai 2019. URL http://journals.openedition.org/ries/2958; DOI : 10.4000/ries.2958

Ce document a été généré automatiquement le 1 mai 2019.

(C) Tous droits réservés 


\title{
La minorité acadienne au Canada atlantique
}

\author{
Rodolphe M.J. Toussaint
}

1 Pays d'immigration, le Canada est caractérisé par une très grande diversité ethnique et culturelle, reflet des origines diverses de ses citoyens. Au gré des mouvements migratoires, des groupes en provenance de France et des îles britanniques sont d'abord venus s'ajouter aux Amérindiens, premiers occupants du territoire. Par la suite, d'autres régions européennes ont fourni d'importants contingents d'immigrants qui se sont répartis dans les diverses provinces d'un pays en pleine construction. Depuis les vingt dernières années, les nouveaux immigrants arrivent surtout des pays asiatiques et du Moyen-Orient, des Caraïbes et de l'Amérique latine ${ }^{1}$ Pour décrire cette diversité d'origines ethnique, linguistique, culturelle et aussi sociale de la population canadienne, Porter ${ }^{2}$ utilisait l'expression, décriée depuis, de « mosaïque verticale ».

2 Le présent article traite de l'identité, de la diversité culturelle et d'éducation chez les Acadiens, un groupe minoritaire de langue française établi sur le continent nordaméricain depuis les premières colonisations européennes au XVII siècle. Quatre cents ans plus tard, les Acadiens des trois provinces maritimes constituent une minorité linguistique canadienne qui a su développer et conserver une identité particulière.

3 Comment cette identité a-t-elle pu se maintenir dans le grand ensemble canadien, sans se fondre dans la majorité linguistique anglophone environnante?

$4 \mathrm{Au}$ fil des siècles, des événements extérieurs, d'abord européens puis continentaux, ont exercé une influence marquée sur le développement et la survivance du groupe ethnoculturel acadien sur le sol nord-américain. Nous évoquerons d'abord les conditions de son installation. Nous considérerons ensuite les caractéristiques identitaires et le rôle de l'éducation dans le maintien de cette identité. 


\section{La présence acadienne en Amérique}

Agriculteurs venus de l'Ouest de la France à partir des années 1600, les Acadiens fondent les premières colonies françaises en Amérique dans la baie de Fundy ${ }^{3}$, avant-postes d'où les explorateurs partent pour conquérir des territoires vers le sud et faire commerce de fourrures avec les premiers occupants amérindiens, Micmacs, Malécites et Abénaquis.

Distribution des Acadiens francophones des provinces maritimes

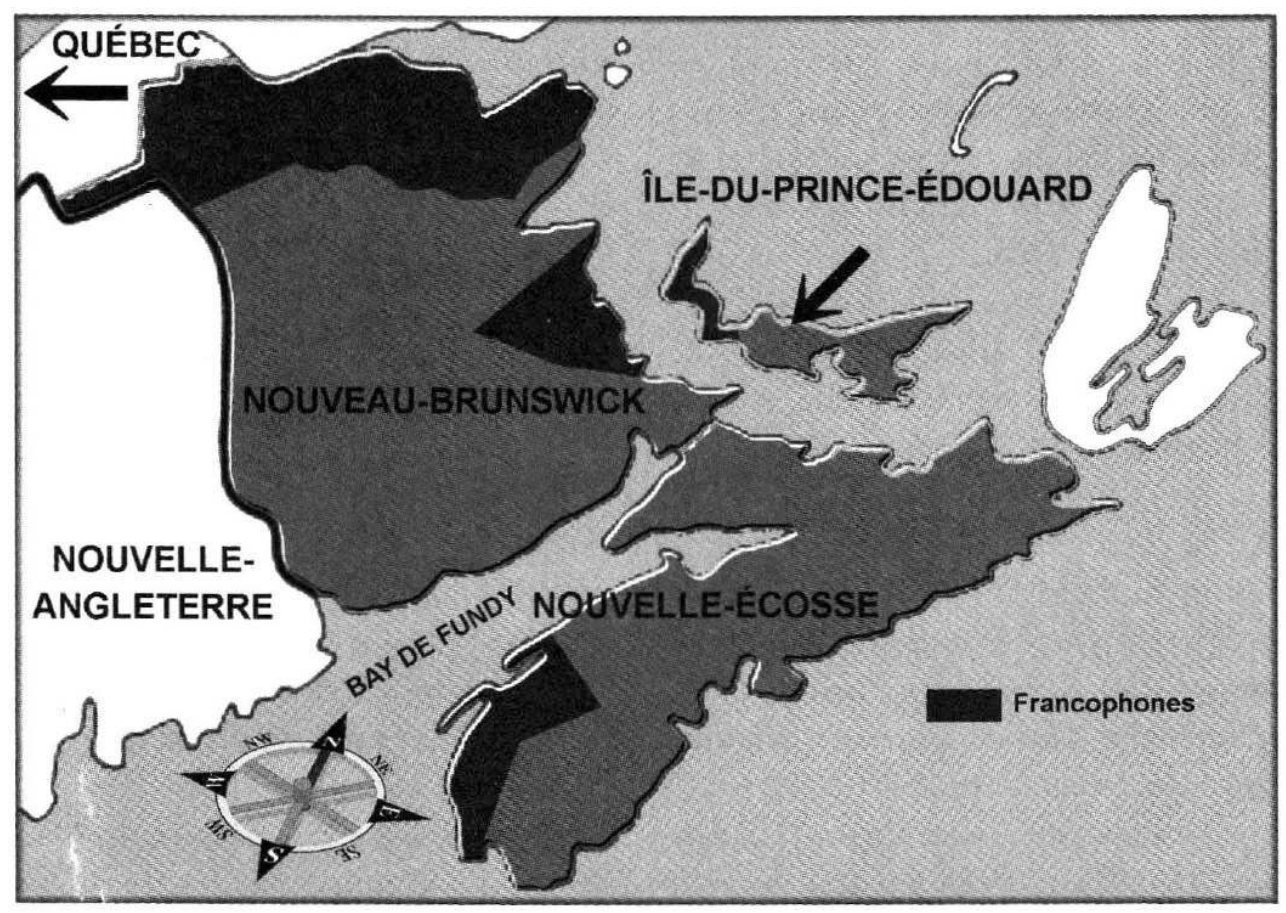

En 1710, la population de l'Acadie est estimée à 1700 habitants comparée aux 16000 de Nouvelle-France dans le golfe et sur les rives du Saint-Laurent et aux 115000 habitants de Nouvelle-Angleterre sur la côte atlantique ${ }^{4}$. Le traité d'Utrecht, qui met fin aux conflits européens entre la France et l'Angleterre, fera de l'Acadie une colonie anglaise en 1713, avec possibilité pour les Acadiens de «jouir du libre exercice de la religion catholique et de conserver leurs terres et tenures et d'en jouir sans aucun trouble ${ }^{5} »$. Signés en Europe, les traités exigeaient d'eux allégeance à la couronne britannique et respect des lois et coutumes anglaises.

7 Sur le terrain, ces signatures ne seront cependant pas honorées puisque dès 1749 arrivent des colons du Massachusetts et le gouverneur britannique décide de la redistribution des riches terres agricoles de la vallée d'Annapolis. Les Acadiens, qui opposent une résistance très peu passive à devenir sujets britanniques, sont dépouillés du droit de propriété et de celui de l'usage du français dans la vie publique. Se produisent alors une série d'événements qui, de juillet 1755 jusqu'à l'année 1763, entraînent leur dispersion et leur déportation vers l'Angleterre, la France et les colonies anglaises. Rares sont ceux qui évitent la déportation en se réfugiant dans les forêts et sur la côte est du NouveauBrunswick. Le déplacement forcé de ces 12000 personnes, considéré comme une nécessité militaire à l'époque ${ }^{6}$, aura réussi à freiner pendant près d'un siècle l'établissement francophone dans cette région d'Amérique. Paradoxalement, ces événements 
contribueront à doter cette population dispersée d'une identité proprement acadienne qui fait l'objet de ce texte.

8 La révolution américaine de 1776 créera un influx de trente mille "Loyalistes", population anglophone anti-insurrectionniste et loyale à l'Angleterre. La couronne britannique leur concédera des terres au sud du Nouveau-Brunswick et dans l'île du CapBreton. La société acadienne, nettement minoritaire, se replie sur elle-même, érige un système d'autosuffisance et perd, pour presqu'un siècle, la mainmise sur sa destinée.

Racontée diversement par des historiens, des poètes, des romanciers ou des essayistes, « le grand dérangement » de 1755 constitue l'élément déterminant de l'identification des Acadiens à une collectivité particulière, américaine plutôt qu'européenne. Le renouveau de l'Acadie se fera d'abord à partir de la mémoire collective d'une aventure qui, dans sa première étape, avait duré près de 160 ans. La reconstitution du peuplement acadien prendra encore un siècle à se réaliser de sorte qu'en 1867, à la naissance de la confédération canadienne, cette population compte environ 80000 habitants dans les provinces maritimes. Entre-temps, elle s'était dotée d'instruments collectifs de développement culturel et social : collèges et couvents, clergé, institutions économiques, journaux d'opinion?

\section{Minorité culturelle et identité}

10 L'identité ethnique d'un groupe se manifeste par « l'appropriation subjective de symboles et d'emblèmes culturels ainsi que par la perception d'une origine commune et d'un sentiment de continuité $»^{8}$, toutes caractéristiques qui contribuent à le distinguer de tout autre groupe. À part la perpétuation biologique et les valeurs culturelles communes, le groupe se « donne un espace de communication et d'interaction ${ }^{9} »$. En milieu minoritaire, ces caractéristiques sont contrastées par les sentiments d'aliénation et d'exclusion éprouvés face au groupe majoritaire qui prend allure ou fonction d'oppresseur.

\section{La géographie}

11 Les Acadiens se sont toujours identifiés à un territoire particulier dont les limites sont constituées des lieux où les habitants s'identifient comme tels et participent en tant que groupe homogène à la vie sociale, économique et culturelle. Diverses conceptions de la territorialité explorées par les géographes ${ }^{10}$ proposent une Acadie historique (Est du Canada, le Maine aux USA et la Gaspésie au Québec), généalogique (Amérique du Nord, Antilles, Amérique du sud, France), opérationnelle (le Nouveau-Brunswick, la NouvelleÉcosse et l'île-du-Prince-Édouard) ${ }^{11}$, prospective (comtés et paroisses civiles acadiennes du N.-B.). Dans les années soixante-dix, un parti politique, le Parti acadien, s'était donné pour objectif l'obtention d'une autonomie territoriale et éventuellement la création d'une province nouvelle épousant les contours de cette Acadie prospective ${ }^{12}$.

\section{La langue}

12 La caractéristique emblématique et culturelle essentielle de l'identité acadienne est l'utilisation du français comme langue première dans les interactions entre individus. Cette utilisation a aussi valeur symbolique de reconnaissance dans le groupe et de différenciation vers l'extérieur. Souvent considérée comme exotique hors d'Acadie, la 
langue acadienne est distincte du français québécois, autant dans l'accent que par l'utilisation de mots, d'expressions et de formes syntaxiques anciennes. Elle différencie aussi les régions acadiennes et l'oreille un tant soit peu exercée peut discerner le parler néo-brunswickois, de celui de la Nouvelle-Écosse ou de la Louisiane ${ }^{13}$.

Divers facteurs contribuent généralement aux transferts linguistiques, à l'assimilation et à un échange d'identité vers le groupe majoritaire. La concentration ou la dispersion de la population acadienne dans un territoire donné influence la rétention ou la perte du français comme langue d'usage. La proximité de la langue dominante et la tolérance entre groupes conduisent aussi graduellement à un usage uniquement privé et familial du français. L'exogamie linguistique constitue un facteur d'assimilation et l'on constate que seul un couple mixte sur cinq utilise le français comme langue de communication.

Dans les régions où se côtoient Acadiens et anglophones, cette langue subit les assauts constants de la langue majoritaire. La sociologue Muriel Roy cite ce titre d'un long métrage louisianais «C'est nice de parler des deux manières » qui révèle l'ampleur du processus d'accommodation et de glissement vers l'autre langue.

D'ailleurs, plus les secteurs économique et technologique se modernisent et s'ouvrent vers l'extérieur, et plus se pose pour ces francophones de l'Amérique « le problème de la redéfinition de la place du français et de l'anglais partout dans la société acadienne ${ }^{14}$ ». Sauf dans les institutions proprement acadiennes et dans les secteurs gouvernementaux néo-brunswickois de l'éducation ou de la santé, il est difficile, sinon impossible, de fonctionner dans une langue autre que l'anglais. Une clause de l'actuelle constitution canadienne précise que le Nouveau-Brunswick est une province officiellement bilingue, l'anglais et le français ayant valeur égale devant la loi et dans l'usage gouvernemental.

Dans la population d'origine ethnique française ou de langue maternelle française, les transferts linguistiques vers le groupe majoritaire anglophone sont assez élevés sauf au Nouveau-Brunswick, désormais centre géographique de l'Acadie où le taux d'assimilation, sans pour autant diminuer fortement, semble s'être stabilisé autour de $2 \%{ }^{15}$. La langue française y a gardé un niveau de prestige qui assure la «vitalité ethnolinguistique » des Acadiens de cette province.

Comme le précisent les chercheurs Landry, Allard, Hogg et Abrams ${ }^{16}$, "le groupe ethnolinguistique aura un statut d'autant plus élevé qu'il contrôle sa destinée économique, a une haute estime de soi, est fier de son passé et utilise une langue de réputation internationale ».

\section{La démographie}

Particulièrement dans le cas du groupe minoritaire, la taille relative de la population doit permettre de résister à la pression démographique et à la puissance d'attraction des groupes majoritaires environnants. Un facteur d'importance pour éviter le changement d'identité consiste donc en la capacité de rétention sur un territoire délimité d'un seuil minimal d'individus partageant les mêmes caractéristiques identitaires. 
Évolution de la population d'origine ethnique française dans les maritimes

\begin{tabular}{|c|c|rr|rr|cr|r|}
\hline \multicolumn{2}{|c|}{} & & \multicolumn{2}{|c|}{$\begin{array}{c}\text { Origine ethnique } \\
\text { française }\end{array}$} & $\begin{array}{c}\text { Langue maternelle } \\
\text { française }\end{array}$ & $\begin{array}{c}\text { Français, langue } \\
\text { d'usage }\end{array}$ & $\begin{array}{c}\text { Population } \\
\text { de la province }\end{array}$ \\
\hline & 1871 & 44907 & $15,7 \%$ & & & & & 285594 \\
Nouveau- & 1881 & 56635 & $17,6 \%$ & & & & & 321233 \\
Brunswick & 1971 & 235025 & $37,0 \%$ & 215725 & $33,9 \%$ & 199070 & $31,4 \%$ & 634560 \\
& 1981 & 251070 & $36,1 \%$ & 251940 & $33,3 \%$ & 216585 & $31,1 \%$ & 696403 \\
& 1991 & 235010 & $32,5 \%$ & 250175 & $34,6 \%$ & 225950 & $31,2 \%$ & 723900 \\
\hline & 1871 & 32833 & $8,5 \%$ & & & & & 387800 \\
Nouvelle- & 1881 & 41219 & $9,4 \%$ & & & & & 440572 \\
Écosse & 1971 & 80215 & $10,2 \%$ & 39335 & $4,9 \%$ & 27220 & $3,4 \%$ & 788960 \\
& 1981 & 71350 & $8,4 \%$ & 35690 & $4,2 \%$ & 24450 & $2,9 \%$ & 847442 \\
& 1991 & 55310 & $6,1 \%$ & 39425 & $4,4 \%$ & 23120 & $2,6 \%$ & 899940 \\
\hline & 1871 & 9250 & & & & & & \\
Îles-du-Prince- & 1881 & 10751 & $9,9 \%$ & & & & & 108891 \\
Édouard & 1971 & 15325 & $13,7 \%$ & 7365 & $6,6 \%$ & 4405 & $3,9 \%$ & 111640 \\
& 1981 & 14770 & $12,1 \%$ & 5915 & $4,8 \%$ & 3725 & $3,0 \%$ & 122506 \\
& 1991 & 11845 & $9,1 \%$ & 6285 & $4,8 \%$ & 3165 & $2,4 \%$ & 129765 \\
\hline
\end{tabular}

Source : Roy (1993) et Statistiques Canada.

19 La croissance de la population d'origine ethnique française a aussi été étroitement liée aux événements historiques cités plus haut. Alors que le pourcentage des «parlant français » semble s'être maintenu au Nouveau-Brunswick à hauteur de $30 \%$ de la population depuis les trente dernières années, ce taux fléchit en Nouvelle-Écosse et à l'île-du-Prince-Édouard. La décroissance est accentuée par un faible taux de natalité, l'émigration de personnes actives vers les grands centres économiques ${ }^{17}$ et la grande difficulté d'inclusion de nouveaux immigrants dans la population acadienne.

\section{Idéologie}

Jusqu'aux années 1960, l'expression de « survivance » paraît dans tous les travaux traitant du phénomène acadien. La conservation du fait français est prônée dans toutes les institutions et surtout celles où œuvre le clergé catholique. L'idéologie dominante passait alors par le discours, seule arme dont disposait l'élite acadienne pour tenter de conquérir un espace social et briser l'isolement culturel ${ }^{18}$. La résistance s'organisait alors autour de «l'Église, l'école et l'hôpital ", discours résolument conservateur et antimoderniste ${ }^{19}$.

La laïcisation des institutions d'éducation, la création d'universités francophones modernes (Moncton au Nouveau-Brunswick et Ste-Anne en Nouvelle-Écosse) et l'accession aux leviers économiques caractérisent la modernité acadienne. Cette société se trouve cependant confrontée à des forces externes apparemment antithétiques aux représentations qu'elle se donnait d'elle-même. La mondialisation et son corollaire, l'utilisation d'une langue internationale, la situation géographique de l'Acadie, en périphérie des grands centres économiques, entraînent une ouverture sur l'extérieur qui la fait se remettre en question et porte les germes d'une nouvelle « dispersion». 


\section{L'école en Acadie, gardienne de l'identité culturelle}

Dès les premiers établissements, l'éducation a joué un rôle primordial dans ce qu'il est convenu d'appeler «la survivance acadienne $»^{20}$. Les éducateurs sont d'abord des Jésuites et des Récollets venus de France pour "catéchiser et instruire » colons et amérindiens. Après la déportation et le retour en terre d'Acadie, le système éducatif est plutôt précaire et la responsabilité de l'éducation francophone repose sur des maitres ambulants et quelques écoles privées francophones, paroissiales pour la plupart.

Entre 1864 et 1877, dans la foulée des mouvements de laïcisation de l'éducation en Europe et en Amérique, les diverses chambres législatives des provinces maritimes adoptent des lois instituant l'éducation pour tous les enfants dans une école publique neutre et unilingue anglaise ${ }^{21}$. Une taxe obligatoire devait être levée sur les propriétés pour financer le fonctionnement des nouvelles écoles. Ces lois précisent aussi l'obligation d'obtention d'un brevet pour les enseignants, diplôme inaccessible - ou tout au moins difficile d'obtention - aux enseignants francophones, la langue d'enseignement dans les écoles de formation pédagogique étant uniquement l'anglais. Du coup, deux cent cinquante classes françaises fermaient leurs portes. Catastrophiques pour le développement de la population francophone et le maintien de sa présence en terre d'Amérique, une nouvelle vicissitude s'ajoutait à une histoire déjà assez bouleversée ${ }^{22}$.

4 Les évêques catholiques, voulant surtout protéger les institutions religieuses d'éducation, défendent à leurs ouailles de faire éduquer les enfants dans les écoles publiques, à leurs yeux dominées par les protestants. À mentionner que ces évêques étaient irlandais d'origine, anglophones et faisaient partie eux-mêmes d'une minorité en situation défensive.

Catholiques, les Acadiens se trouvent confrontés au dilemme de désobéir à la hiérarchie et de fréquenter une école publique gouvernementale où les seuls livres en français agréés en 1875 seront deux manuels de lecture bilingue : «Le premier livre de lecture First Reading Book » et «Le second livre de lecture - The Second Reading Book ».

S'ensuit une période de création d'institutions privées, collèges et couvents, dans les paroisses francophones avec l'aide de congrégations québécoises ${ }^{23}$. La double taxation est le lot des contribuables francophones qui choisissent d'y éduquer leurs enfants, aucun subside gouvernemental ne leur étant accordé.

7 L'Acte de l'Amérique du Nord britannique de 1867, acte constitutionnel de la Confédération canadienne, reconnaît pourtant l'usage de la langue française dans toutes les provinces. Le champ de l'éducation y est reconnu de compétence exclusive de chaque législature provinciale, sans possibilité d'intervention pour la législature fédérale.

28 Dans les années qui suivirent, les résultats de ces politiques ont été désastreux pour le système des écoles publiques francophones; l'abandon scolaire atteindra un sommet de $90 \%$ dès les six premières années de scolarité. La formation pédagogique est minimale et à l'école normale de Fredericton, fondée en 1848, un «département français » sera institué en 1887 qui accorde le brevet de deuxième classe après un an d'études et le brevet de troisième classe après trois mois. Seul le brevet de première classe confirmait la permanence du droit d'enseigner à plein salaire.

La situation reflète l'attitude générale de l'époque d'indifférence face à l'école publique, l'élite intellectuelle anglophone choisissant généralement les écoles privées pour 
l'éducation de ses enfants ${ }^{24}$. Il faudra attendre les années d'après la Seconde Guerre mondiale pour qu'il y ait renouvellement d'intérêt pour une éducation de qualité dans le système public.

\section{L'Acadie moderne}

30 Les francophones avaient développé entre 1864 et 1963 un réseau privé d " "établissements de langue française conduisant... à des baccalauréats ${ }^{25}$ ». Ce système a été remplacé par deux universités francophones publiques qui s'occupent de la formation post-secondaire en Nouvelle-Écosse et au Nouveau-Brunswick.

31 L'université de Moncton octroie des diplômes de deuxième cycle dans toutes ses facultés et de troisième cycle en études françaises et en psychologie. Une trentaine de groupes et d'instituts, subventionnés par les entreprises privées ou gouvernementales, y développent une recherche de pointe dans divers domaines des arts, des sciences humaines, des sciences sociales et des sciences appliquées.

32 Après de nombreuses années d'incertitude quant à la formation des enseignants, une école normale française est créée en 1968, qui sera intégrée en 1973 dans une structure facultaire de l'université de Moncton ${ }^{26}$.

33 En 1964, le ministère de l'Éducation du Nouveau-Brunswick est subdivisé en deux parties autonomes, une anglophone, l'autre francophone. Les francophones obtiennent pleine autorité sur les programmes d'enseignement dans leurs écoles primaires et secondaires. Dans les deux autres provinces, des sections particulières des ministères ont droit de regard sur les curriculums des écoles francophones.

34 Après tant de combats pour le droit à une identité propre et à la diversité, les Acadiens font encore preuve d'une étonnante capacité à résister et à rebondir. Les institutions d'éducation ont suscité un foisonnement d'activités intellectuelles, culturelles, sociales et économiques. Il n'est que de constater la richesse de la littérature contemporaine ${ }^{27}$, les succès internationaux d'entrepreneurs installés en Acadie, la reconnaissance sociale et politique qui fait que l'actuel gouverneur du Canada et le Premier ministre du NouveauBrunswick sont des Acadiens.

Le défi est cependant d'adapter cette école aux réalités modernes de l'ouverture au monde, de la qualité des moyens de communication et de la place des Acadiens -malgré leur faible nombre-dans le débat canadien actuel.

\section{NOTES}

1. 1 K. Krótki et C. Reid, "Demography of Canadian Population by Ethnic Group », in: Berry et Laponce (éd.), Ethnicity and Culture in Canada: the Research Landscape, Toronto, University of Toronto Press, 1994, 579 p. S. Majhanovich, Canadian Multiculturalism at the Crossroads: Implications for Education. 9th World Congress of Comparative and International Education, 1-6 juillet 1996, Sydney, Australia. 
2. John Porter, The Vertical Mosaic: an Analysis of Social Class and Power in Canada, Toronto, University of Toronto Press, 1965, $626 \mathrm{p}$.

3. Sainte-Croix et Port-Royal ont été les premiers postes permanents de colonisation française dans la baie française, actuelle baie de Fundy.

4. A. Bernard, Histoire de l'Acadie, Moncton, L'Évangéline Ltée, 1938, p. 22.

J. Daigle, «L'Acadie de 1604 à 1763, synthèse historique », in : Daigle (dir.), L'Acadie des Maritimes : études thématiques des débuts à nos jours, Moncton, Université de Moncton, Chaire d'études acadiennes, 1993, p. 22.

5. E. Le Blanc, Les Acadiens, Les éditions de. l'Homme, 1963, p. 19.

6. La déportation faisait bien partie des mœurs militaires et sera invoquée ou utilisée indifféremment par les armées coloniales, française ou anglaise.

7. Le premier numéro du Moniteur acadien paraît le 8 juillet 1867 et ce journal sera publié jusqu'en 1926.

8. G. De Vos, «Ethnic pluralism », p. 15-47, in: Romanucci-Ross et DeVos (Ed.) Ethnic Identity. Creation, Conflict, and Accommodation, Walnut Creek, CA, AltaMira Press, 1996.

Driedger, The Ethnie Factor: Identity in Diversity, Toronto, McGraw-Hill Ryerson, 1989.

9. F. Barth, "Les groupes ethniques et leurs frontières ", p. 203-249, in : Poutignat et StreiffFenart, Théories de l'ethnicité, Paris, PUF, 1995.

10. 11 J.C. Vernex, Les francophones du Nouveau-Brunswick: géographie d'un groupe ethnoculturel minoritaire, Paris, Librairie Honoré Champion, 1978, thèse Université de Lyon II, 1975.

A. Bérubé, Concevoir un manuel de géographie du Nouveau-Brunswick, Thèse de Ph.D, Université Laval, 1990.

11. Voir la carte.

12. R. Ouellette, Le Parti acadien: de la fondation à la disparition, 1972-1982, Moncton, N.-B. Chaire d'études acadiennes, 1992, $119 \mathrm{p}$.

13. Lire Herméningilde Chiasson (artiste acadien), "Trente identités sur un nombre illimité », p. 267-289, in: Létourneau, La question identitaire au Canada francophone, Ste-Foy, Presses de l'Université Laval, 1994.

14. L. Péronnet, « La situation du français en Acadie », p. 467-503, in : Daigle (éd.), op. cit.

15. Voir tableau.

16. R. Allard et R. Landry, «Ethnolinguistic Vitality ", numéro spécial, Journal of the Sociology of Language, 1992.

M. Hogg et D. Abrams, Social identifications: a social psychology of intergroup relations and group processes, New York, Routledge, 1988.; M. Hogg, The social psychology of group cohesiveness: from attraction to social identity, New York, New York University Press, 1992.

17. L'historien M. Roy évalue à 40000 le nombre d'Acadiens de 15-35 ans qui ont émigré de 1970 à 1980.

18. 19 J.-P. Hautecoeur, L'Acadie du discours: pour une sociologie de la culture acadienne, Québec, Presses de l'Université Laval, 1975, $351 \mathrm{p}$.

19. M. Roy, L'Acadie perdue, Montréal, Québec/Amérique, 1978, 203 p.

M. Roy, L'Acadie des origines à nos jours, Montréal : Québec/Amérique, 1989, 340 p.

20. A. Bernard, Histoire de la survivance acadienne, Montréal, Les Clercs de Saint-Viateur, 1935.

21. G. Couturier-Le Blanc, A. Godin et A. Renaud, L'enseignement français dans les Maritimes, 1604-1992, p. 543-585, in : Daigle (dir.), op. cit.; A. Deveau, Notre héritage acadien. L'histoire du groupe ethnique acadien de 1755 à nos jours, Pointe-de-l'Église, Imprimerie de l'Université Sainte-Anne, chap. 28, p. 371-385.

22. En 1875, à Caraquet, il y aura même un mouvement de révolte et de refus de l'école neutre, avec mort d'homme. 
23. À sa fondation, le collège St-Joseph dirigé par des francophones sera bilingue et servira à l'éducation des Acadiens et des Irlandais catholiques sur demande de Mgr Sweeney, l'évêque de St-Jean au Nouveau-Brunswick.

24. C. Phillips, "The development of public school Systems; The Provinces", p. 214-233, The Development of Education in Canada, Toronto, Gage, 1957, $626 \mathrm{p}$

25. G. Couturier-Le Blanc et al., op. cit., p. 576.

26. E. Picot, Les écoles normales du Nouveau-Brunswick 1848-1973, Fredericton, N.-B., 1974, 157 p.

A. Bailey, "The Professional Preparation of Teachers for the Schools of the Province of New Brunswick, 1784 to 1964 », Part II: 1900-1964, Ontario Journal of Educational Research, 7(3), 231-239, 1965.

27. M. Maillet, Bibliographie des publications de l'Acadie des provinces maritimes. 1609-1995, Moncton, Les éditions d'Acadie, 1997, 555 p. ; Histoire de la littérature acadienne. De rêve en rêve, Moncton, Les éditions d'Acadie, 1983, $262 \mathrm{p}$.

\section{RÉSUMÉS}

Au Canada, l'identité ethno-culturelle des Acadiens, minorité de langue française, doit à l'école sa survie et sa vitalité. Cette école doit encore s'adapter aux réalités du monde moderne pour que les Acadiens, malgré leur petit nombre, continuent à jouer un rôle dans le grand ensemble canadien.

\section{INDEX}

Index géographique : Canada

Mots-clés : minorité culturelle, minorité linguistique, langue française, politique éducative, politique linguistique

\section{AUTEUR}

\section{RODOLPHE M.J. TOUSSAINT}

Professeur, département des sciences de l'éducation, Université du Québec à Trois-Rivières, Québec, Canada 